\title{
Design of Laser Guiding Target for Shield
}

\author{
Li Wei $^{1, a,{ }^{*}}$, Yujing Guo ${ }^{1}$, Chenguang Zhao ${ }^{1}$
}

\author{
${ }^{1}$ Department of Mechanical \& Electrical Engineering, Tangshan College, Hebei Tangshan, China \\ aysuweili@163.com \\ *corresponding author
}

Keywords: laser guiding, attitude angle, shield.

\begin{abstract}
In the process of tunnel excavation, in order to ensure the error between the direction of travel and the designed tunnel axis within required range, the relationship between the position of the shield and the designed axis of the tunnel should be monitored and measured in real time. The laser displacement detection target based on embedded technology is an important part of the shield laser guidance. It can perform the measurement of the position and angle of the shield by the internal CCD camera, tilt sensor and prism. The laser detection target is fixing on the shield. The total station can calculate the spatial position coordinates of prism which is on the laser target by visual reference. The laser target can get the shield attitude angle. The shield tail incision center coordinates can be obtained by coordinate conversion.
\end{abstract}

\section{Introduction}

Transportation industry has developed rapidly in recent years, urban subway project in many second-tier cities have started to become an important way of the national travel. Because of the complicated geological conditions in different regions, underground excavation construction exists various technical problems. The shield tunneling construction which is not affected by the weather, don't affect the ground transportation, and has little influence on the ground buildings and underground pipelines is idely used.

Shield tunneling is a kind of full mechanized construction method in the construction of concealed excavation method. The shield machine is in the underground, through the shield shell and shield segments prevent to the collapse of the tunnel surrounding rock. At the same time, the cutting device is used to excavate the soli moved outside by machinery, jacking by jack in the back and then assembling precast concrete segments forming structure of tunnel ${ }^{[1]}$.

Shield driving should be strictly in accordance with the designed route. At present, there are kinds of methods for the guiding system, such as three prisms guiding system, double prisms guiding system and target guiding system ${ }^{[2]}$. In this paper, a tight encapsulation of laser arget guiding system is designed. It can perform real-time measurement of shield attitude angle by the optical sensor total station and rearview prisms, which composing the complete laser guiding system.

\section{Construction of shield guiding system}

The shield guiding system is mainly composed of a total station, a reference prism and a laser target, as shown in Figure 1. The coordinates of visual reference prism is known. Total station emits a laser beam to the reference prism and bounces back. The elevation and coordinate angle of the total station can be obtained, which is the absolute coordinates of the total station.

After the position of the total station is determined, the laser device is rotated and the laser light search the laser target. There is a cube-corner retroreflector facing the total station is fixed on the laser target. The cube-corner retroreflector is a tetrahedron maded up of three mutually perpendicular reflective surface. Its most obvious characteristic is that the light incident from the bottom after the reflection of the three vertical surfaces, the direction of the emergent light is opposite. The emergent light is reflected back to the laser by the cube-corner retroreflector, and the 
total station can determine the position of the laser target .

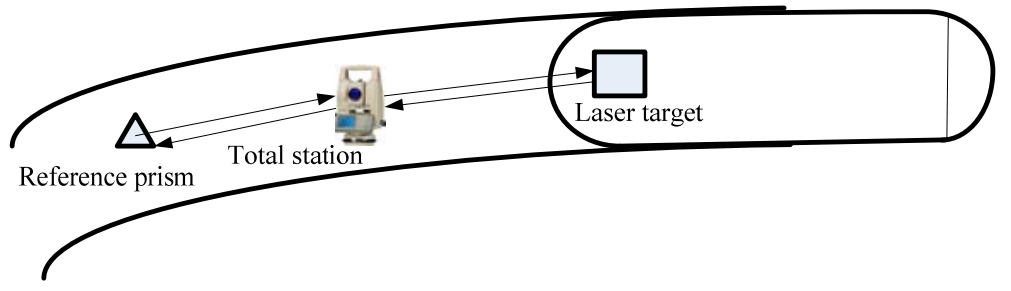

Figure 1 The composition of guiding system.

\section{Measurement Principle of laser target}

\subsection{Structure of laser target}

The laser target mainly includes a tilt sensor, CCD, cube-corner retroreflector, embedded circuit board and varous interfaces, as shown in Figure 2.

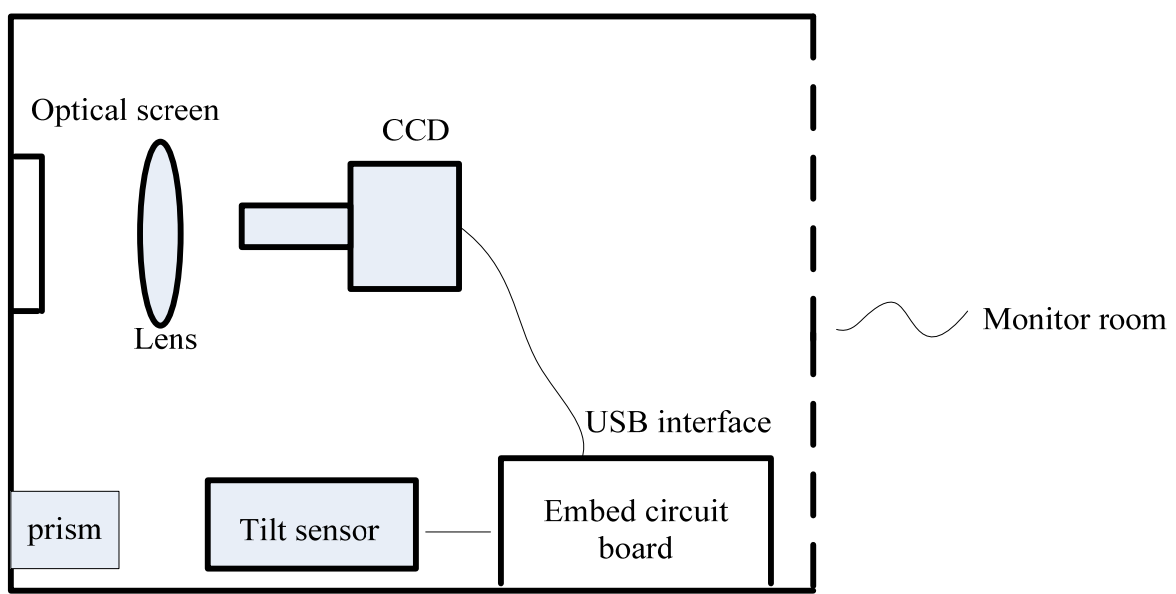

Figure 2 The schematic diagram of laser detection target.

The laser from the total station is irradiated on the target screen. The spot can be formed on the CCD receiving surface which is at the focal plane of the lens. The position of the spot is related to the incident angle of the laser and it can be obtained by CCD. The spot image is transferred to the embedded processing module and the image processing toolbox of openCV soft is used to calculate the spot position. The position can be used to determine the azimuth and elevation angle of the laser target. The rolling angle of the laser target is measured by the tilt sensor. So the spatial attitude and position coordinates of the laser target fixed on the shield are all available, which can determine the attitude and position information of the shield machine.

\subsection{Measurement of Rolling angle}

The rolling angle is caused by the reaction force between the cutter and the soil layer and rotational axis is its own axis. As the shield rotation in gravity plane around its own axis, the rolling angle of shield machine is changed. So the rolling angle can be obtained by the change of gravity ${ }^{[4]}$, as shown in Figure 3.

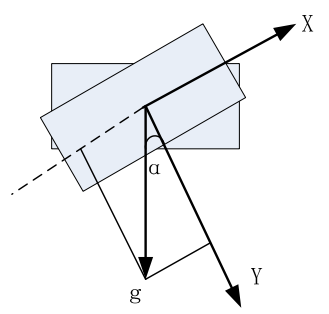

Figure 3 Measurement principle diagram of the rolling Angle.

Assuming that $\mathrm{X}$ and $\mathrm{Y}$ are the two axes of the tilt sensor, gx and gy is components of gravity 
at the axis $\mathrm{X}$ and $\mathrm{Y}$. According to the geometric relationship in figure 3, the formula (1) is get as follow,

$$
\alpha=\operatorname{actan}(\mathrm{gx} / \mathrm{gy})
$$

According to the measurement principle of inclinometer, the rolling angle of shield is measured by the horizontally plated inclinometer and the the angle data is transmitted to CPU by serial port.

\subsection{Azimuth Angle and horizontal Angle measurement}

The azimuth angle and horizontal angle of the laser target coordinate system are determined by the location of the incident laser spot from CCD. Then the attitude angle in the absolute coordinate system can be obtained by coordinate transformation. So the key is how to get the laser spot center coordinates. Laser target has the seal structure, however, due to the limitations of the production process, there are still a small amount of stray light inside the target. At the same time, the non-uniformity of the incident light will cause the uneven brightness of the spot. So we need to use digital image processing technology to smooth and sharp processing. ARM9 embedded chip is selected as processing CPU and OpenCV3.0, which is in the Linux environment programming, is used to perform the vision algorithm.

The image preprocessing includes image acquisition, digitization, gray level transformation and spatial filtering, image enhancement and image sharpening, etc. According to the characteristics of circular spot in this system, the smoothing processing of the original image as the same time keeping the gray information of the spot edge is key. Finally the image center coordinates is determined. The basic process as shown in Figure 4.

\begin{tabular}{|c|c|c|c|}
\hline $\begin{array}{c}\text { Image } \\
\text { Acquisition }\end{array}$ & $\begin{array}{l}\text { Morphological } \\
\text { filter }\end{array}$ & $\begin{array}{l}\text { Coordinates } \\
\text { caculation }\end{array}$ & $\begin{array}{l}\text { Attitude angle } \\
\text { caculation }\end{array}$ \\
\hline
\end{tabular}

Figure 4 The processing of spot image.

The methods of image smoothing processing mainly include the domain average method, low-pass filtering method and multi image average method. The laser spot image is complex and single filtering method can not meet the requirements. So the morphological filtering method is adopted for processing. Dilation and erosion are the most basic morphological operations. Two basic operations can be used to achieve opening and closing operations, morphological gradient, top hat transform, black hat transform, and other more advanced morphological transformation. The opening operation can be used to eliminate small objects, and separate objects in fine point. The closing operation can enhance the dark details smaller than the structural elements, and the extent of weakening and enhancement depends on the size of these features relative to the structural element ${ }^{[5]}$.

Because of the internal defects of dark areas in the spot, the closing operation is selected to perform Morphological filtering operation, The key statement is $\mathrm{dst}=\mathrm{close}(\mathrm{src}$,element $)=$ erode (dilate(src,element)).

After the image filtering, centric method is used to obtain the coordinates of the spot center, as shown in formula (2).

$$
(x, y)=\left(\frac{\sum_{\mathrm{i}=1}^{\mathrm{M}} \sum_{\mathrm{j}=1}^{\mathrm{N}} \mathrm{i} \times \mathrm{f}(\mathrm{i}, \mathrm{j})}{\sum_{\mathrm{i}=1}^{\mathrm{M}} \sum_{\mathrm{j}=1}^{\mathrm{N}} \mathrm{f}(\mathrm{i}, \mathrm{j})}, \frac{\sum_{\mathrm{i}=1}^{\mathrm{M}} \sum_{\mathrm{j}=1}^{\mathrm{N}} \mathrm{j} \times \mathrm{f}(\mathrm{i}, \mathrm{j})}{\sum_{\mathrm{i}=1}^{\mathrm{M}} \sum_{\mathrm{j}=1}^{\mathrm{N}} \mathrm{f}(\mathrm{i}, \mathrm{j})}\right.
$$

$\mathrm{M}$ and $\mathrm{N}$ is the boundary of the ROI(Region of interest) of the image, $f(i, j)$ is the gray value of the pixel pot(x,y).

\section{Conclusion}

In the process of tunnel excavation, it is necessary to monitor and measure the relationship between the position of the shield and the designed axis of the tunnel in order to ensure the error is limited to the allowable range. In the moving process of shield, the main guiding parameters include the cutter center coordinates of shield machine and three angles, namely rolling angle, pitch angle and the horizontal angle. The attitude and position of the shield is determined by the three angle measurement and the coordinates of the center of the head shield. In this paper it is to study a set of 
laser guiding system for measuring the position and angle of the shield. It mainly solves two key problems. Firstly the measurement system is designed, which includes three parts to built the absolute coordinate and provide the light source. Secondly the laser target is designed, which includes the prism to locate the target position, the image sensor to get the spot. This system is simple construction, convenient calculation and high precision.

\section{References}

[1] Ye Zhiming. (2016)Introduction to Civil Engineering. Higer Education Press: Beijing, 115

[2] Bi Xiaowei, Research on key technology for measuring shield machine's position and orientation . Shanghai Jiao Tong University Shanghai, P.R.China.2010: 2

[3] Liu Guanlan, Li Jianzhou. Analysis of angle measurement error of the cube cornerretro-reflector with oblique incident laser beam. Geotechnical Investigation \& Surveying,2017:45

[4] Shao Tao. Key Technology for Laser Target Used in Shield Attitude Measurement. Huazhong University of Science \& Technology Wuhan, P.R.China.2012:9

[5] Mao xingyun, Leng xuefei. OpenCV3 Programming Primer. Electronic Industry Press: Beijing, $198-200$ 